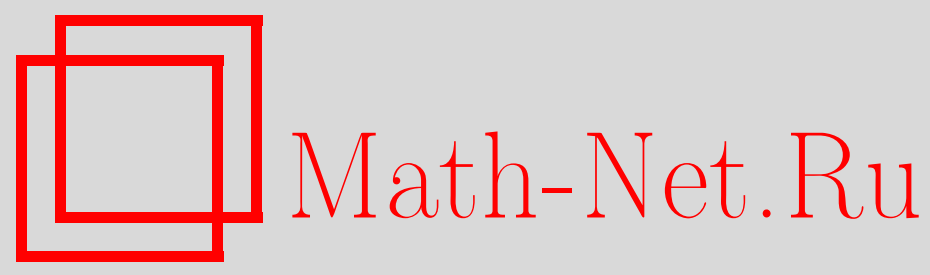

Я. Н. Нужин, Об одном вопросе М. Кондера, Матем. заметки, 2001, том 70, выпуск 1, 79-87

DOI: https://doi.org/10.4213/mzm721

Использование Общероссийского математического портала Math-Net.Ru подразумевает, что вы прочитали и согласны с пользовательским соглашением http://www . mathnet.ru/rus/agreement

Параметры загрузки:

IP : 54.237 .206 .68

26 апреля 2023 г., 14:59:59

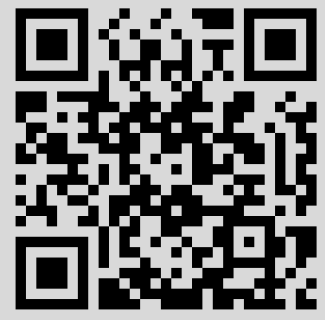


УДК 519.4

\section{ОБ ОДНОМ ВОПРОСЕ М. КОНДЕРА}

\section{Я.Н. Нужин}

Доказано, что $S L_{3}(\mathbb{Z})$ не порождаются двумя элементами порядков 2 и 3. Библиограффия: 6 названий.

Основным результатом работы является

ТЕорема. Специальная линейная группа размерности 3 над кольиом иельх чисел $S L_{3}(\mathbb{Z})$ не порождается двумя әлементами порядков 2 и 3.

Эта теорема ${ }^{1}$ дает отрицательный ответ на следующий вопрос М. Кондера из [1, вопрос 14.49].

Является ли группа $S L_{3}(\mathbb{Z})$ факторәруппой әруппы $P S L_{2}(\mathbb{Z}) ?$ Так как модулярная группа изоморфна свободному произведению двух ииклических групп порядков 2 $u 3$, то вопрос в том, порохдается ли группа $S L_{3}(\mathbb{Z})$ двумя әлементами порядков $2 u 3$.

Изоморфизм групшы $P S L_{2}(\mathbb{Z})$ и свободного произведения двух циклических групп порядков 2 и 3 был установлен Р. Фрике и $\Phi$. Клейном в 1890 году [2]. Групшы, порождаемые двумя элементами порядков 2 и 3 , будем называть $(2,3)$-порожденными. Очевидно, группа $S L_{2}(\mathbb{Z})$ не $(2,3)$-порожденная, так как она содержит единственную инволюцию, которая является центральной. Такая же и группа $S L_{4}(\mathbb{Z})$ в силу ее гомоморфизма на $S L_{4}\left(\mathbb{Z}_{2}\right) \simeq A_{8}$ и следуюшего результата $\Gamma$. А. Миллера [3]. Знакопеременная группа $A_{n}, n \geqslant 5$, тог да и только тогда $(2,3)$-порожденная, когда $n \neq 6,7,8$. С другой стороны, в [4] показано, что при $n \geqslant 28$ группа $S L_{n}(\mathbb{Z})$ является $(2,3)$-порожденной. Таким образом, остается открытым вопрос: будет ли $(2,3)$-порожденной группа $S L_{n}(\mathbb{Z})$ при $5 \leqslant n \leqslant 27$ ? Кажется правдоподобным, что и в этих случаях ответ будет положительным. Потому что, как правило, при $n \geqslant 5$ для групшы $S L_{n}(K)$ над каким-то кольцом $K$ уже начинается общая ситуация.

Конечно, вопрос М. Кондера можно поставить еше шире: какие групшы Шевалле над кольцом цельх чисел являются $(2,3)$-группами?

Основной результат статьи анонсировался в [5].

Работа выполнена при финансовой поддержке Российского фонда фундаментальных исследований, грант № 99-01-01256.

${ }^{1}$ Когда статья была уже подготовлена, В. Д. Мазуров сообщил автору, что аналогичньй результат получили итальянские математики Ш. Тамбурини и П. Цукка (препринт). 
1. Определения и предварительные результаты. Далее всюду $\mathbb{Z}$ и $\mathbb{Q}$ - кольцо целых и соответственно поле рациональных чисел.

Лемма 1. Все әлементы порядка 3 из $S L_{3}(\mathbb{Q})$ сопряэсены в $G L_{3}(\mathbb{Q})$.

ДокАЗАТЕЛЬСТво. Покажем, что любой элемент $B=\left(b_{i j}\right)$ порядка 3 из $S L_{3}(\mathbb{Q})$ сопряжен в $G L_{3}(\mathbb{Q})$ с матрицей

$$
\beta=\left(\begin{array}{lll}
0 & 0 & 1 \\
1 & 0 & 0 \\
0 & 1 & 0
\end{array}\right)
$$

При сопряжении матрицы $B$ мономиальными $(0,1)$-матрицами транзитивно переставляются элементы матрицы $B$ на всех позициях $(i, j)$ при $i \neq j$. Поэтому либо $B$ - диагональная матрица, либо с точностью до сопряжения мономиальной $(0,1)$-матрицей $b_{13} \neq 0$. Так как мультипликативная группа поля рациональных чисел не содержит элементов порядка 3 , то первьй случай не возможен.

Пусть $b_{13} \neq 0$. В этом случае с точностью до сопряженности диагональным элементом из $G L_{3}(\mathbb{Q})$ можно считать, что $b_{13}=1$, т.е.

$$
B=\left(\begin{array}{lll}
b_{11} & b_{12} & 1 \\
b_{21} & b_{22} & b_{23} \\
b_{31} & b_{32} & b_{33}
\end{array}\right)
$$

Сопрягая сейчас матрицу $B$ последовательно трансвекциями

$$
\left(\begin{array}{ccc}
1 & 0 & 0 \\
-b_{23} & 1 & 0 \\
0 & 0 & 1
\end{array}\right), \quad\left(\begin{array}{ccc}
1 & 0 & 0 \\
0 & 1 & 0 \\
-b_{33} & 0 & 1
\end{array}\right), \quad\left(\begin{array}{ccc}
1 & 0 & 0 \\
0 & 1 & 0 \\
0 & b_{12} & 1
\end{array}\right), \quad\left(\begin{array}{ccc}
1 & 0 & 0 \\
0 & 1 & 0 \\
b_{11} & 0 & 1
\end{array}\right),
$$

получаем, что с точностью до сопряжения $B$ имеет вид

$$
\left(\begin{array}{lll}
0 & 0 & 1 \\
a & d & 0 \\
c & b & 0
\end{array}\right)
$$

Здесь и далее при сопряжении какой-то матрицей $X$ на обратную матрицу $X^{-1}$ умножаем справа. Так как любая матрица порядка 3 из $S L_{3}(\mathbb{Q})$ имеет жорданову нормальную форму вида $\operatorname{diag}\left(\theta, 1, \theta^{2}\right)$, где $\theta$ - первообразньй корень третьей степени из единицы, то $\operatorname{tr} \beta=0$ и, в частности, $d=0$. Таким образом, из условия $\beta^{3}=1$ получаем, что и $c=0$. Легко проверить, что при $d=c=0$ матрица $B$ имеет порядок 3 только при $a b=1$. Сопрягая теперь матрицу $B$ диагональной матрицей $\operatorname{diag}\left(1, a, a^{-1}\right)$, получим матрицу $\beta$. Лемма доказана.

Как обьчно, под ииркулянтом (циркулянтной матрицей размерности 3) будем понимать следующую матрицу:

$$
T=T(t, u, v)=\left(\begin{array}{ccc}
t & u & v \\
v & t & u \\
u & v & t
\end{array}\right)
$$

Если матрица $\beta$ такая же, как и в доказательстве леммы 1 , то имеет место следующее представление:

$$
T=t \beta^{3}+u \beta^{2}+v \beta
$$

Из этого представления следует, что все циркулянты над каким-либо ассоциативным коммутативным кольцом с единицей составляют коммутативную полугрупшу, а множество всех обратимых циркулянтов является грушой. 
ЛЕмма 2. Пусть $T=T(t, u, v)-$ ииркулянт, а $T^{\prime}=T^{\prime}\left(t^{\prime}, u^{\prime}, v^{\prime}\right)$ - его обратная ииркулянтная матрица. Тогда

$$
(t+u+v)\left(t^{\prime}+u^{\prime}+v^{\prime}\right)=1
$$

ДокАЗАТЕЛЬСТво. Сложив коэффициенты в первой строке у произведения $T T^{\prime}$, получим искомое равенство. Лемма доказана.

ЛЕмма 3. Пусть $T=T(t, u, v)$ - ииркулянт над полем комплексных чисел $c$ условием $t+u+v=1 u T^{\prime}=T^{\prime}\left(t^{\prime}, u^{\prime}, v^{\prime}\right)$ - его обратная ииркулянтная матрица. $\Pi$ Псть

$$
\alpha=\left(\begin{array}{ccc}
-1 & 0 & 0 \\
a & 1 & b \\
0 & 0 & -1
\end{array}\right)
$$

причем сумма $a+b$ является рациональным числом, отличным от $-2, u T^{\prime} \alpha T \in$ $S L_{n}(\mathbb{Q})$. Тогда все числа $t, u, v, a, b$ являются рациональными.

ДоКАЗАТЕЛЬСТВо. Положим

$$
\begin{gathered}
T^{\prime} \alpha T=\left(a_{i j}\right), \quad i, j=1,2,3, \\
s_{i}=a_{i 1}+a_{i 2}+a_{i 3}, \quad i=1,2,3 .
\end{gathered}
$$

По лемме $2 t+u+v=t^{\prime}+u^{\prime}+v^{\prime}=1$. Простые вычисления показывают, что

$$
s_{1}=(a+b+2) u^{\prime}-1, \quad s_{2}=(a+b+2) t^{\prime}-1, \quad s_{3}=(a+b+2) v^{\prime}-1 .
$$

По условию леммы все $s_{i}$ должны быть рациональными числами и $a+b+2 \neq 0$. Отсюда каждое из чисел $t, u, v$ рациональное. Теперь из условия леммы вытекает, что $\alpha \in S L_{n}(\mathbb{Q})$, а следовательно, числа $a$ и $b$ также рациональные. Лемма доказана.

ЛЕмма 4. Пусть $a, b$ - комплексные числа, причем $a+b \neq-2, u$ әруппа $M$, порохсденная двумя матрицами

$$
\alpha=\left(\begin{array}{ccc}
-1 & 0 & 0 \\
a & 1 & b \\
0 & 0 & -1
\end{array}\right), \quad \beta=\left(\begin{array}{lll}
0 & 0 & 1 \\
1 & 0 & 0 \\
0 & 1 & 0
\end{array}\right)
$$

сопряжена в $G L_{3}(C)$ с подгруппой из $S L_{3}(\mathbb{Q})$. Тогда $M$ сопряжена в $G L_{3}(\mathbb{Q}) c$ той же подгруппой из $S L_{3}(\mathbb{Q})$ и, в частности, числа а, $b$ рачиональные.

ДокАЗАТЕЛЬСТво. По условию леммы существуют невырожденная комплексная матрица $A=\left(a_{i j}\right)$ и матрицы $B=\left(b_{i j}\right)$ и $C=\left(c_{i j}\right)$ из $S L_{3}(\mathbb{Q})$ такие, что

$$
\begin{aligned}
& \alpha A=A C, \\
& \beta A=A B .
\end{aligned}
$$


Запишем (2) более подробно в виде

$$
\left(\begin{array}{lll}
a_{31} & a_{32} & a_{33} \\
a_{11} & a_{12} & a_{13} \\
a_{21} & a_{22} & a_{23}
\end{array}\right)=\left(\begin{array}{lll}
a_{11} & a_{12} & a_{13} \\
a_{21} & a_{22} & a_{23} \\
a_{31} & a_{32} & a_{33}
\end{array}\right)\left(\begin{array}{lll}
b_{11} & b_{12} & b_{13} \\
b_{21} & b_{22} & b_{23} \\
b_{31} & b_{32} & b_{33}
\end{array}\right) .
$$

Матричное равенство (3) задает систему линейных однородных уравнений с целочисленными коэффициентами относительно неизвестных $a_{i j}$. В следующей таблице первая строка задает упорядоченные неизвестные $a_{i j}$, а остальные девять составляют основную матрицу системы (3):

\begin{tabular}{|ccc|ccc|ccc|}
\hline$a_{11}$ & $a_{12}$ & $a_{13}$ & $a_{21}$ & $a_{22}$ & $a_{23}$ & $a_{31}$ & $a_{32}$ & $a_{33}$ \\
\hline$b_{11}$ & $b_{21}$ & $b_{31}$ & 0 & 0 & 0 & -1 & 0 & 0 \\
$b_{12}$ & $b_{22}$ & $b_{32}$ & 0 & 0 & 0 & 0 & -1 & 0 \\
$b_{13}$ & $b_{23}$ & $b_{33}$ & 0 & 0 & 0 & 0 & 0 & -1 \\
\hline-1 & 0 & 0 & $b_{11}$ & $b_{21}$ & $b_{31}$ & 0 & 0 & 0 \\
0 & -1 & 0 & $b_{12}$ & $b_{22}$ & $b_{32}$ & 0 & 0 & 0 \\
0 & 0 & -1 & $b_{13}$ & $b_{23}$ & $b_{33}$ & 0 & 0 & 0 \\
\hline 0 & 0 & 0 & -1 & 0 & 0 & $b_{11}$ & $b_{21}$ & $b_{31}$ \\
0 & 0 & 0 & 0 & -1 & 0 & $b_{12}$ & $b_{22}$ & $b_{32}$ \\
0 & 0 & 0 & 0 & 0 & -1 & $b_{13}$ & $b_{23}$ & $b_{33}$ \\
\hline
\end{tabular}

В блочном виде основную матрицу можно записать в виде

$$
\left(\begin{array}{ccc}
B & O & -E \\
-E & B & O \\
O & -E & B
\end{array}\right)
$$

Умножив ее слева последовательно на матрицы

$$
\left(\begin{array}{ccc}
O & E & O \\
O & O & E \\
E & O & O
\end{array}\right), \quad\left(\begin{array}{ccc}
E & O & O \\
O & E & O \\
B & O & E
\end{array}\right), \quad\left(\begin{array}{ccc}
E & O & O \\
O & E & O \\
O & B^{2} & E
\end{array}\right)
$$

получим

$$
\left(\begin{array}{ccc}
-E & B & O \\
O & -E & B \\
O & O & O
\end{array}\right)
$$

Таким образом, ранг основной матрицы равен 6 , т.е. фундаментальная система решений однородной системы (3) состоит из трех векторов, которые будем записьвать в виде $(3,3)$-матриц.

По лемме 1 существует рациональное решение системы (3)

$$
A=\left(a_{i j}\right)
$$

причем $\operatorname{det} A \neq 0$. С другой стороны, матрицы $\beta A$ и $\beta^{2} A$ тоже являются решениями системы (3) и, более того, вместе с $A$ они составляют базис пространства решений этой 
системы. Действительно, если $t A+u \beta A+v \beta^{2} A=0$ для каких-то $t, u, v \in C$, то, учитьвая, что $A$ - невырожденная матрица, получаем

$$
T=\left(\begin{array}{lll}
t & v & u \\
u & t & v \\
v & u & t
\end{array}\right)=O
$$

Итак, по условию леммы сушествует комплексная невырожденная матрица, удовлетворяющая уравнениям (1) и (2). По доказанному вьше она представляется в виде $T A$, где $T$ - циркулянтная невырожденная матрица, а $A$-невырожденная матрица с рациональными коэффициентами. С точностью до умножения на скалярную матрицу можно считать, что $t+u+v=1$. В этом случае по лемме 3 из уравнения (1) вытекает, что подгрупша $M$ сопряжена в $G L_{3}(\mathbb{Q})$ с подгруппй $\langle B, C\rangle$ из $S L_{3}(\mathbb{Q})$ и, в частности, числа $a$ и $b$ рациональные. Лемма доказана.

Лемма 5. Пусть подгруппа $H$ из $S L_{n}(\mathbb{Z})$ порождается множеством матрии, у которых сумма по все столбиам (строкам) нечетна. Тогда $H$ - собственная подгруппа әруппы $S L_{n}(\mathbb{Z})$.

ДокАЗАТЕЛЬСТво. Образ подгрупшы $H$ при гомоморфизме $S L_{n}(\mathbb{Z})$ на $S L_{n}\left(\mathbb{Z}_{2}\right)$, индуцированном кольцевьм гомоморфизмом $\mathbb{Z}$ на $\mathbb{Z}_{2}$, является приводимой подгрупой, так как $H$ стабилизирует вектор $(1,1, \ldots, 1)$. Поэтому $H$ - собственная подгруппа в $S L_{n}(\mathbb{Z})$. Лемма доказана.

Пусть $K$-произвольное ассоциативное коммутативное кольцо с единицей. Набор его идеалов $\sigma=\sigma_{i j}$ называется ковром идеалов, если

$$
\sigma_{i k} \sigma_{k j} \subseteq \sigma_{i j}, \quad i, j, k=1,2, \ldots, n
$$

Множество матриц

$$
\left\{x \in S L_{n}(K) \mid x_{i j} \equiv \delta_{i j} \bmod \sigma_{i j}\right\}
$$

является группой, которая назьвается ковровой подгруппой, определяемой ковром $\sigma$ (см., например, [6, с. 145]).

ЛЕмма 6. Пусть $a, b-$ рациональные числа $u a+b=2$. Положим

$$
\alpha=\left(\begin{array}{ccc}
-1 & 0 & 0 \\
a & 1 & b \\
0 & 0 & -1
\end{array}\right), \quad \beta=\left(\begin{array}{ccc}
0 & 0 & 1 \\
1 & 0 & 0 \\
0 & 1 & 0
\end{array}\right)
$$

Пусть существует невырохсденная матрица $R$ из $G L_{3}(\mathbb{Q})$ такая, что

$$
M=R^{-1}\langle\alpha, \beta\rangle R \subseteq S L_{3}(\mathbb{Z})
$$

Тогда $M$ являемся собственной подгруппой группь $S L_{3}(\mathbb{Z})$. 
ДоКАЗАТЕЛЬСТВО. Очевидно, что матрица $R$ может быть представлена в виде произведения $D A$, где $D$ - скалярная матрица, а $A=\left(a_{i j}\right)$ - матрица с цельми коэффициентами. Поэтому по условию леммы сушествуют матрицы $B=\left(b_{i j}\right)$ и $C=\left(c_{i j}\right)$ из $S L_{3}(\mathbb{Z})$ и взаимно простые целые числа $p$ и $q$ такие, что

$$
\begin{aligned}
& \left(\begin{array}{ccc}
-1 & 0 & 0 \\
\frac{p}{q} & 1 & \frac{2 q-p}{q} \\
0 & 0 & -1
\end{array}\right)\left(\begin{array}{ccc}
a_{11} & a_{12} & a_{13} \\
a_{21} & a_{22} & a_{23} \\
a_{31} & a_{32} & a_{33}
\end{array}\right)=\left(\begin{array}{ccc}
a_{11} & a_{12} & a_{13} \\
a_{21} & a_{22} & a_{23} \\
a_{31} & a_{32} & a_{33}
\end{array}\right)\left(\begin{array}{lll}
c_{11} & c_{12} & c_{13} \\
c_{21} & c_{22} & c_{23} \\
c_{31} & c_{32} & c_{33}
\end{array}\right), \\
& \left(\begin{array}{lll}
0 & 0 & 1 \\
1 & 0 & 0 \\
0 & 1 & 0
\end{array}\right)\left(\begin{array}{lll}
a_{11} & a_{12} & a_{13} \\
a_{21} & a_{22} & a_{23} \\
a_{31} & a_{32} & a_{33}
\end{array}\right)=\left(\begin{array}{lll}
a_{11} & a_{12} & a_{13} \\
a_{21} & a_{22} & a_{23} \\
a_{31} & a_{32} & a_{33}
\end{array}\right)\left(\begin{array}{lll}
b_{11} & b_{12} & b_{13} \\
b_{21} & b_{22} & b_{23} \\
b_{31} & b_{32} & b_{33}
\end{array}\right) .
\end{aligned}
$$

Пусть $q$ нечетное. Через $\mathbb{Z}[1 / q]$ обозначим расширение кольца $\mathbb{Z}$ с помощью элемента $1 / q$. В силу нечетности $q$ кольцевой гомоморфизм $\mathbb{Z} \rightarrow \mathbb{Z}_{2}$ индуцирует групповой гомоморфизм $\varphi: S L_{3}(\mathbb{Z}[1 / q]) \rightarrow S L_{3}(2)$. Если подгруппа $M$ совпадает с $S L_{3}(\mathbb{Z})$, то ее образ при гомоморфизме $\varphi$ должен быть изоморфен $S L_{3}(2)$, что невозможно в силу приводимости подгрупшы $\varphi(\langle\alpha, \beta\rangle)$, которая стабилизирует вектор $(1,1,1)$. Таким образом, при нечетном $q$ подгруппа $M$ собственная.

Далее предполагаем, что $q$ четно, а $p$ нечетно. С точностью до умножения на скалярную матрицу можно считать, что хотя бы один из коэффишиентов $a_{i j}$ нечетен. Уравнения (3) и (6) эквивалентны, а с учетом таблишы (4) они дают следующее утверждение: если в какой-то строке матрицы $A$ все элементы четные, то все элементы матрицы $A$ четные, а так как матрица $A$ имеет нечетный коэффициент, то в каждой ее строке найдется хотя бы по одному нечетному элементу. С другой стороны, из (5) следует, что сумма

$$
a_{1 i} \frac{p}{q}+a_{2 i}+a_{3 i} \frac{2 q-p}{q}, \quad i=1,2,3,
$$

является целым числом. Поэтому

$$
p\left(a_{1 i}-a_{3 i}\right)=m_{i} q, \quad i=1,2,3,
$$

для некоторого целого числа $m_{i}$. Отсюда в силу четности $q$ числа $a_{1 i}$ и $a_{3 i}, i=1,2,3$, одновременно либо четные, либо нечетные.

Пусть все $a_{11}, a_{12}, a_{13}$ нечетные. Тогда $a_{31}, a_{32}, a_{33}$ также являются нечетными. Из $(5)$ и (6) следует, что у матриц $C$ и $B$ суммы по всем столбцам нечетные. Поэтому по лемме 5 матришы $C$ и $B$ не могут порождать всю группу $S L_{3}(\mathbb{Z})$.

Пусть $a_{11}, a_{12}$ четные, а $a_{13}$ нечетное. Тогда $a_{31}, a_{32}$ также являются четными, а $a_{33}$ нечетное. Из (5) и (6) следует, что $c_{31}, c_{32}, b_{31}, b_{32}$ четные. Следовательно, группа $\langle B, C\rangle$ лежит в собственной ковровой подгрупше групшы $S L_{3}(\mathbb{Z})$, определяемой ковром

$$
\left(\begin{array}{ccc}
\mathbb{Z} & \mathbb{Z} & \mathbb{Z} \\
\mathbb{Z} & \mathbb{Z} & \mathbb{Z} \\
2 \mathbb{Z} & 2 \mathbb{Z} & \mathbb{Z}
\end{array}\right)
$$

Аналогично рассматриваются другие два случая, когда $a_{12}, a_{13}$ четные, а $a_{11}$ нечетное, или $a_{11}, a_{13}$ четные, а $a_{12}$ нечетное. В этих случаях группа $\langle B, C\rangle$ лежит в собственных ковровых подгруппах группы $S L_{3}(\mathbb{Z})$, определяемых соответственно коврами

$$
\left(\begin{array}{ccc}
\mathbb{Z} & 2 \mathbb{Z} & 2 \mathbb{Z} \\
\mathbb{Z} & \mathbb{Z} & \mathbb{Z} \\
\mathbb{Z} & \mathbb{Z} & \mathbb{Z}
\end{array}\right), \quad\left(\begin{array}{ccc}
\mathbb{Z} & \mathbb{Z} & \mathbb{Z} \\
2 \mathbb{Z} & \mathbb{Z} & 2 \mathbb{Z} \\
\mathbb{Z} & \mathbb{Z} & \mathbb{Z}
\end{array}\right) .
$$


Остается рассмотреть три случая, когда в первой строке по два нечетных элемента. Все они подобны, поэтому рассмотрим один их них, например, когда $a_{11}, a_{12}$ нечетные.

Итак, пусть $a_{11}, a_{12}$ нечетные, а $a_{13}$ четное. Определим, какой вид имеют матрицы $B$ и $C$. Из (5) и (6) вытекает, что в парах $c_{11}$ и $c_{21}, c_{12}$ и $c_{22}, b_{11}$ и $b_{21}, b_{12}$ и $b_{22}$ элементы имеют разную четность, а в парах $c_{13}$ и $c_{23}, c_{13}$ и $c_{23}$ - одинаковую. Отсюда при

$$
L=\left(\begin{array}{lll}
1 & 0 & 0 \\
1 & 1 & 0 \\
0 & 0 & 1
\end{array}\right)
$$

матрицы $L C L^{-1}$ и $L B L^{-1}$ лежат в ковровой подгрупе, определяемой ковром

$$
\left(\begin{array}{ccc}
\mathbb{Z} & \mathbb{Z} & \mathbb{Z} \\
2 \mathbb{Z} & \mathbb{Z} & 2 \mathbb{Z} \\
\mathbb{Z} & \mathbb{Z} & \mathbb{Z}
\end{array}\right) .
$$

Поэтому подгруппа $M$ является собственной в групше $S L_{3}(\mathbb{Z})$. Лемма доказана.

2. Доказательство основной теоремы. Пусть $\alpha$ - произвольная инволюция, а $\beta$ - произвольньй элемент порядка 3 из $S L_{3}(\mathbb{Z})$. Положим

$$
M=\langle\alpha, \beta\rangle .
$$

Покажем, что в любом случае подгруппа $M$ является собственной подгруппй группы $S L_{3}(\mathbb{Z})$.

Используя жорданову нормальную форму, несложно получить два следуюших утверждения:

1) любая инволюция из $S L_{3}(\mathbb{Z})$ приводится сопряжением матрицей из $G L_{3}(C)$ к виду $\operatorname{diag}(-1,-1,1)$ и, в частности, $\operatorname{tr} \alpha=-1$

2) любой элемент порядка 3 из $S L_{3}(\mathbb{Z})$ приводится сопряжением матрищей из $G L_{3}(C)$ к виду $\operatorname{diag}\left(\theta, 1, \theta^{2}\right)$, где $\theta$ - примитивньй корень степени 3 из 1 , и, в частности, $\operatorname{tr} \beta=0$.

Таким образом, можно считать, что $\alpha=\left(a_{i j}\right)$ для некоторых комплексных чисел $a_{i j}$, a $\beta=\operatorname{diag}\left(\theta, 1, \theta^{2}\right)$.

Возможны следуюшие четыре случая:

1) $a_{12}=a_{13}=0$

2) $a_{12}=0, a_{13} \neq 0$

3) $a_{12} \neq 0, a_{13}=0$

4) $a_{12} a_{13} \neq 0$.

Очевидно, в первом случае подгруппа $M$ приводима. Во втором и в третьем случаях из условия $\alpha^{2}=1$ получаем $a_{32}=0$ и соответственно $a_{23}=0$, и снова в обоих случаях $M-$ приводимая подгруппа. Рассмотрим последний случай.

Пусть $a_{12} a_{13} \neq 0$. С точностью до сопряженности диагональньп элементом из $G L_{3}(C)$ можно считать, что

$$
a_{11}=a, \quad a_{22}=b, \quad a_{12}=k, \quad a_{13}=m,
$$


где $a, b$ - фиксированные, а $k, m$ - произвольные ненулевые комплексные числа. Выразим через $a, b, k, m$ остальные элементы $a_{i j}$ матрицы $\alpha$, используя условие $\alpha^{2}=1$.

Так как $\operatorname{tr} \alpha=0$, то

$$
a_{33}=-1-a-b \text {. }
$$

Далее равенство $a_{i 1} a_{1 j}+a_{i 2} a_{2 j}+a_{i 3} a_{3 j}=\delta_{i j}$, где $\delta_{i j}-$ символ Кронекера, кратко будем обозначать через $(i, j)$. Равенство $(1,2)$ есть $a k+k b+m a_{32}=0$; отсюда

$$
a_{32}=-\frac{k}{m}(a+b)
$$

Подобным образом из $(3,2),(1,3)$ и $(1,1)$ последовательно получаем

$$
a_{31}=-\frac{1}{m}(a+b)(1+a), \quad a_{23}=\frac{m}{k}(1+b), \quad a_{21}=\frac{1}{k}(1+a)(1+b) .
$$

Таким образом,

$$
\alpha=\left(\begin{array}{ccc}
a & k & m \\
\frac{1}{k}(1+a)(1+b) & b & \frac{m}{k}(1+b) \\
-\frac{1}{m}(a+b)(1+a) & -\frac{k}{m}(a+b) & -1-a-b
\end{array}\right) .
$$

Элементарные вычисления показьвают, что

$$
\operatorname{det} \alpha=1+(a+b)(1+a)(1-b) \text {. }
$$

Так как по условию $\operatorname{det} \alpha=1$, то

$$
(a+b)(1+a)(1-b)=0
$$

Если $a=-b$ или $a=-1$, то $M-$ приводимая подгруппа.

Пусть $b=1, a \neq-1$. Тогда

$$
\alpha=\left(\begin{array}{ccc}
a & k & m \\
\frac{2}{k}(1+a) & 1 & \frac{2}{k} m \\
-\frac{1}{m}(1+a)^{2} & -\frac{k}{m}(1+a) & -2-a
\end{array}\right)
$$

Как отмечалось вьше, элементы $k$ и $m$ независимо от $a$ могут принимать любые ненулевые значения. Положим $k=2$, a $m=-1-a$. Тогда

$$
\alpha=\left(\begin{array}{ccc}
a & 2 & -1-a \\
1+a & 1 & -1-a \\
1+a & 2 & -2-a
\end{array}\right)
$$

Если $\theta$ - такой же, как и выше, примитивньй корень степени 3 из 1 , то матрица

$$
B=\left(\begin{array}{ccc}
1 & 1 & \theta^{2} \\
\theta & 1 & \theta \\
\theta^{2} & 1 & 1
\end{array}\right)
$$


обратима и

$$
B^{-1}=\frac{1}{3}\left(\begin{array}{ccc}
1 & -1-\theta & \theta \\
1 & 1 & 1 \\
\theta & -1-\theta & 1
\end{array}\right)
$$

Пусть $D=\operatorname{diag}(1, \theta, 1)$. Тогда

$$
\begin{aligned}
D^{-1} B^{-1} \alpha B D= & \left(\begin{array}{ccc}
-1 & 0 & 0 \\
a\left(\theta^{2}-\theta\right)-\theta^{2}-3 \theta & 1 & a\left(\theta-\theta^{2}\right)-2 \theta^{2}-1 \\
0 & 0 & -1
\end{array}\right) \\
& D^{-1} B^{-1} \beta B D=\left(\begin{array}{lll}
0 & 0 & 1 \\
1 & 0 & 0 \\
0 & 1 & 0
\end{array}\right)
\end{aligned}
$$

причем сумма элементов второй строки матрицы $D^{-1} B^{-1} \alpha B D$ равны 3 . Далее применяем результаты из п. 1.

Очевидно, подгруппа

$$
G=\left\langle D^{-1} B^{-1} \alpha B D, D^{-1} B^{-1} \beta B D\right\rangle
$$

сопряжена в $G L_{3}(C)$ с подгруппой

$$
M=\langle\alpha, \beta\rangle \subseteq S L_{3}(\mathbb{Z})
$$

Поэтому по лемме 4 существует матрица $R \in G L_{3}(\mathbb{Q})$ такая, что

$$
R^{-1} G R=M
$$

Теперь по лемме 6 получаем, что $M-$ собственная подгруппа групшы $S L_{3}(\mathbb{Z})$. Теорема доказана.

\section{СПИСОК ЦИТИРОВАННОЙ ЛИТЕРАТУРЫ}

[1] Мазуров В. Д., Хухро Е. И. Нерешенные вопросы теории групп. Коуровская тетрадь. Изд. 14. Новосибирск, 1999.

[2] Fricke R., Klein F. Vorlesungen über die Theorie der Elliptischen Modulfunktionen. V. 1, 2. Leipzig: Teubner, 1890.

[3] Miller G. A. On the groups generated by two operators // Bull. AMS. 1901. V. 7. P. 424-426.

[4] Tamburini M. C., Wilson J.S., Gavioli N. On the (2,3)-generation of some classical groups I // J. Algebra. 1994. V. 168. P. 353-370.

[5] Нужин Я. Н. Порождающие элементы группы $P S L_{n}(\mathbb{Z}) / /$ IV Международная алгебраическая конференция. Тезисы докл. Новосибирск, 2000. С. 129-130.

[6] Каргаполов М.И., Мерзляков Ю. И. Основы теории групп. М.: Наука, 1982.

Красноярский государственный технический университет

E-mail: nuzhin@fipu.kgtu.runnet.ru 